\title{
Bone Union Rate Following Instrumented Posterolateral Lumbar Fusion: Comparison between Demineralized Bone Matrix versus Hydroxyapatite
}

\author{
Woo Dong Nam, Jemin Yi \\ Department of Orthopaedic Surgery, Kangwon National University College of Medicine, Chuncheon, Korea
}

\begin{abstract}
Study Design: Retrospective study.
Purpose: To compare the union rate of posterolateral lumbar fusion (PLF) using demineralized bone matrix (DBM) versus hydroxyapatite $(\mathrm{HA})$ as bone graft extender.

Overview of Literature: To our knowledge, there has been no clinical trial to compare the outcomes of DBM versus HA as a graft material for PLF.

Methods: We analyzed prospectively collected data from consecutive 79 patients who underwent instrumented PLF. Patients who received DBM were assigned to group $B(n=38)$, and patients who received HA were assigned into group $C(n=41)$. The primary study outcome was fusion rate assessed with radiographs. The secondary outcomes included pain intensity using a visual analogue scale, functional outcome using Oswestry disability index score, laboratory tests of inflammatory profiles and infection rate.

Results: One year postoperatively, bone fusion was achieved in $73 \%$ in group B and $58 \%$ in group C without significant difference between the groups $(p=0.15)$. There were no differences between the groups with respect to secondary outcomes.

Conclusions: DBM would provide noninferior outcomes compared to the HA as a fusion material for PLF, and could be a notable alternative.
\end{abstract}

Keywords: Posterolateral lumbar fusion; Demineralized bone matrix; Hydroxyapatites

\section{Introduction}

Posterolateral lumbar fusion (PLF) is a commonly used surgical intervention to treat degenerative spinal disorders [1]. Successful bony union following lumbar spinal fusion correlates with an improved clinical outcome and better radiologic parameters [2,3]. Pseudarthroses often cause clinical outcomes that are less satisfying than those of bony union and often necessitate further surgery. Although the autogenous iliac bone graft has been the gold standard treatment for obtaining spinal fusion, there can be graft harvest complications, such as chronic donor site pain, infection, fracture, loss of sensation, meralgia paresthetica, and hematomas [4-7]. Allografts are widely used to supplement bone graft materials. However, there are concerns regarding several drawbacks including

\footnotetext{
Received Mar 7, 2016; Revised Apr 13, 2016; Accepted Apr 27, 2016

Corresponding author: Jemin Yi

Department of Orthopaedic Surgery, Kangwon National University Hospital,

156 Baengnyeong-ro, Chuncheon 24289, Korea

Tel: +82-33-258-9209, Fax: +82-33-258-2149, E-mail: firekimdo@gmail.com
} 
fragility or graft fractures, contamination, and disease transmission [4,7-9].

Ideal graft materials or graft substitutes should have three basic biologic attributes of osteogenicity, osteoconductivity, and osteoinductivity [10,11]. Demineralized bone matrix (DBM) has been utilized as a graft substitute because of its osteoinductivity $[4,7,12]$. In addition to its osteoinductivity, DBM serves as an osteoconductive material $[7,12]$. Hydroxyapatite (HA) has been widely utilized for bony regeneration due to several positive physical properties $[4,7]$. HA is chemically similar to human bone mineral and so has excellent compatibility; additionally, HA has superior osteoconductivity $[4,7,8]$.

Use of DBM as a bone graft extender may theoretically provide more potent osteoinductivity than the use of HA. However, to the best of our knowledge, there has been no clinical trial comparing the outcomes of HA and that of DBM as bone graft extender for PLF. Therefore, we analyzed the radiological and clinical outcomes of PLF using DBM (Bonfuse; CG Bio Inc., Seongnam, Korea) compared with HA (Bongros; Bioalpha, Seongnam, Korea), which is widely recognized as an effective fusion material.

\section{Materials and Methods}

\section{Participants}

This study is a retrospective, single institution and comparative analysis of PLF using DBM versus HA as bone graft extenders to supplement local autograft. All clinical data were collected prospectively. The study was approved by the Institutional Review Board of the author's hospital.

The study involved only patients who underwent spinal fusion surgeries from April 2012 to December 2015. The patients fully complied with the following inclusion and exclusion criteria. The inclusion criteria were (1) degenerative lumbar spinal disease or lumbar spondylolytic spondylolisthesis diagnosed using lumbar spine radiographs and magnetic resonance imaging that corresponded to clinical manifestations and physical examinations; (2) one to three segment PLF; (3) age between 40 and 80 years; (4) voluntary participation with written informed consent; and (5) follow-up for 1 year or longer. Those who met any of the following criteria were excluded: (1) fractures, infection, or tumors in the lumbar spine; (2) hemorrhagic disorders, such as hemophilia and thrombocytopenia; (3) follow-up of less than 1 year; and (4) patients judged unsuitable by the principal investigator (the second author).

The type of bone graft was alternately used according to the date. A total of 85 patients underwent PLF during the index period. Six patients were excluded due to less follow up period.

Finally, 79 patients were included in the analysis (38 in group B, DBM; 41 in group C, HA). There were no statistically significant differences of the two groups in demographic characteristics including age, gender, smoking status, height, weight, body mass index, fusion levels, and level of surgery (Table 1).

\section{Surgical procedures and postoperative management protocols}

All patients underwent laminectomies, partial medial facetectomies, transpedicular screw/rod instrumentation, and posterolateral fusion through a posterior midline incision. Resected laminar and facet joint bones were stripped of all soft tissue before being morselized into small pieces and used as autogenous bone grafts in all patients. The average volume of autogenous local bone graft per segment was $7 \mathrm{~mL}$. Great care was taken to ensure adequate decortication of the transverse processes and lateral facet surfaces before the placement of autogenous bone grafts and DBM or HA mixtures. DBM $5 \mathrm{~mL}$ per segment was used for group B and HA $10 \mathrm{~mL}$ per segment was used for group C.

Patients in both groups were treated with the same postoperative protocols. All patients were allowed to ambulate on the first day after surgery. Patients were instructed to keep rigid brace for 3 months. During the postoperative period where oral ingestion was prohibited, patient controlled analgesia was mainly delivered by a fentanyl pump. When oral ingestion resumed, After non per os oral nonsteroidal anti-inflammatory drugs (NSAIDs) and intervenous tramadol were administered routinely during hospitalization. Oral NSAIDs and gabapentin were prescribed at discharge as necessary.

\section{Outcome measures}

The primary outcome was the bone fusion rate at 6 and 12 months after surgery. Arthrodesis was determined to be successful if follow-up radiographs demonstrated a bilateral continuity in the fusion mass between the cephalad 
Table 1. Demographic data

\begin{tabular}{|c|c|c|c|}
\hline Variable & $\begin{array}{c}\text { Group B } \\
(\mathrm{DBM}, \mathrm{n}=38)\end{array}$ & $\begin{array}{c}\text { Group C } \\
(H A, n=41)\end{array}$ & $p$-value \\
\hline Age (yr) & $66.9 \pm 8.3$ & $67.0 \pm 9.1$ & 0.97 \\
\hline Sex (male:female) & $11: 27$ & $13: 28$ & 0.79 \\
\hline Height (cm) & $161.1 \pm 7.1$ & $162.0 \pm 96.1$ & 0.55 \\
\hline Weight (kg) & $58.9 \pm 9.45$ & $61.4 \pm 9.2$ & 0.24 \\
\hline $\mathrm{BMI}\left(\mathrm{kg} / \mathrm{m}^{2}\right)$ & $22.6 \pm 2.4$ & $23.3 \pm 2.5$ & 0.19 \\
\hline Smoking status & & & 0.47 \\
\hline Smoker & $10(34)$ & $14(26)$ & \\
\hline Non-smoker & $28(66)$ & $28(74)$ & \\
\hline Mean BMD $\left(\mathrm{g} / \mathrm{cm}^{2}\right)$ & $-0.841 \pm 1.688$ & $-0.873 \pm 1.688$ & 0.94 \\
\hline Fusion segment & & & 0.77 \\
\hline 1 & $24(63)$ & $23(56)$ & \\
\hline 2 & $11(29)$ & $16(39)$ & \\
\hline 3 & $3(8)$ & $2(5)$ & \\
\hline Level of surgery & & & 0.96 \\
\hline L2-3 & 6 & 3 & \\
\hline L3-4 & 14 & 19 & \\
\hline L4-5 & 29 & 34 & \\
\hline L5-S1 & 6 & 5 & \\
\hline
\end{tabular}

Values in data cells represent mean \pm standard deviation or number (\%).

DBM, demineralized bone matrix; HA, hydroxyapatite; BMI, body mass index; BMD, bone densitometry.

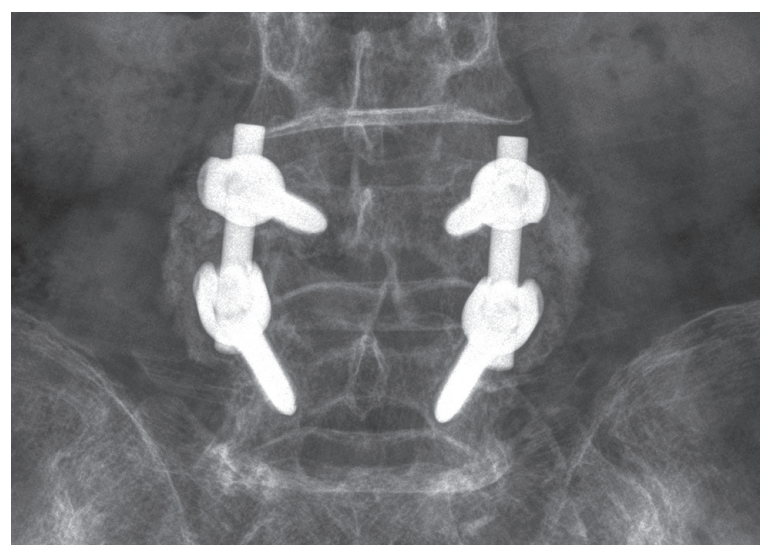

Fig. 1. One-year postoperative radiograph shows bilateral continuity in the fusion mass.

and caudad transverse processes (Fig. 1). Pseudarthrosis was present if there was no continuity in the fusion mass or if lateral flexion-extension radiographs demonstrated greater than $2^{\circ}$ of angular motion or greater than $2 \mathrm{~mm}$ of sagittal motion at the level of the PLF (Fig. 2) [2,3,13]. To determine fusion status of multilevels, all levels not excluding a single level had to show continuity in the fusion mass to be considered as arthrodesis. Radiographs for determining fusion status (anteroposterior, lateral and flexion-extension) were obtained at 3, 6, and 12 months after surgery. The measurement was carried out using a picture archiving and communication system program (Infinitt, Bracknell, Berkshire, UK). All clinical and radiographic assessments were made by examiners other than the surgeons in charge; the assessors were blinded to the clinical results. Radiographs were independently examined by two orthopedic surgeons. Interobserver reliability was 0.72 . If the reported fusion status differed between the examiners, the radiographs were reexamined and a consensus was reached.

Secondary outcomes were (1) intensity of posterior neck pain and pain radiating to the lower extremity based on 10-point visual analogue scale (VAS); (2) Oswestry disability index (ODI); (3) laboratory tests of inflammatory profiles, including erythrocyte sedimentation rate (ESR), C-reactive protein (CRP), and white blood cell count (WBC); and (4) infection rate. These data were collected 

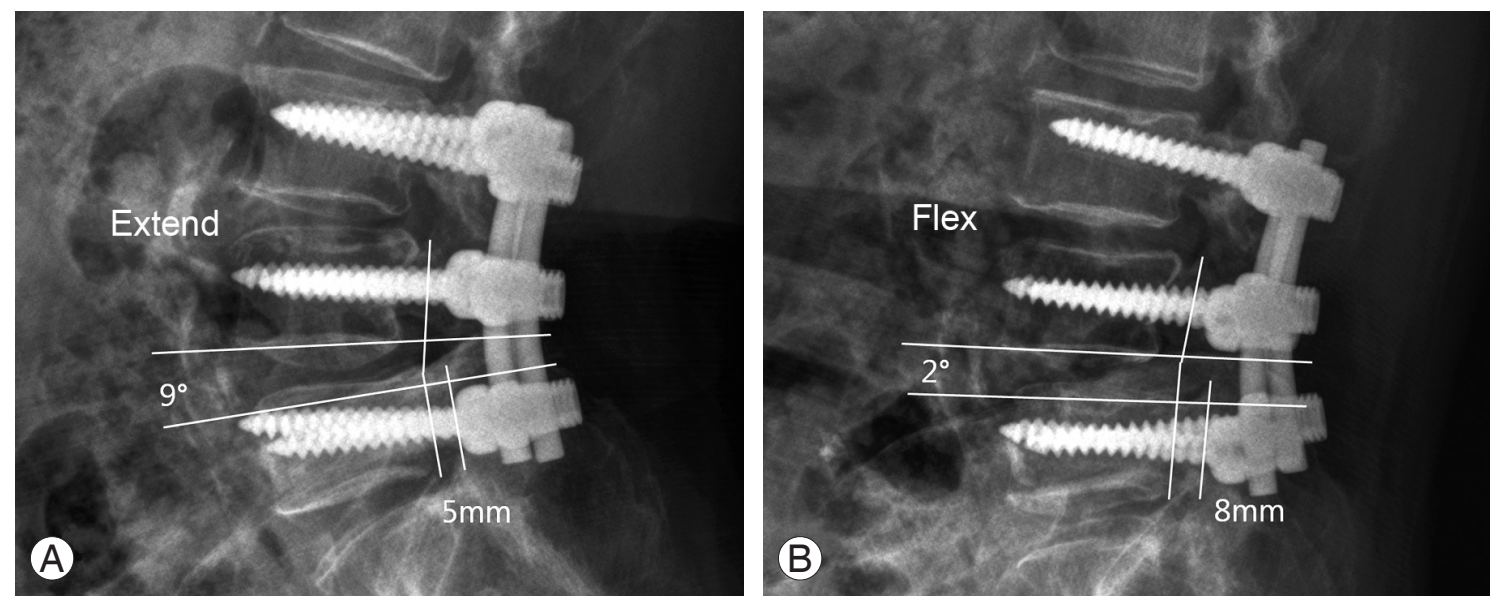

Fig. 2. (A) One-year postoperative extension radiograph demonstrates $5 \mathrm{~mm}$ of subluxation at L4-L5. (B) One-year postoperative extension radiograph demonstrates an increase of suluxation to $8 \mathrm{~mm}$ of subluxation during flexion. This patient's sagittal motion would be $3 \mathrm{~mm}$ and angular motion would be $7^{\circ}$.

and tabulated prospectively by a research coordinator preoperatively and at each follow-up visit at postoperative 1,3 , 6 , and 12 months.

In all patients, bone densitometry was measured prior to surgery using dual-energy X-ray absorptiometry using model QDR-4500 and Delthi devices (Hologic, Waltham, MA, USA). Measurements were obtained by average of L1-4 vertebral body.

\section{Statistical analyses}

The independent Student's $t$ test was used for continuous variables and the chi-square test was used for discrete variables. dBSTAT software ver. 5.0 (Barun Lab, Yongin, Korea) was used for all analyses and a two-sided $p$-value of $<0.05$ was considered statistically significant.

\section{Results}

\section{Primary outcome (fusion rate)}

In radiographs evaluated 3 months after surgery, fusion was achieved in $8 / 38$ patients in group B (21\%) and in $16 / 41$ patients in group C (39\%) (Table 2). The difference was not statistically significant $(p=0.08)$. At 6 months postoperatively, $18 / 38$ patients in group B (47\%) and $25 / 41$ patients in group C (61\%) achieved fusion, without a statistically significant difference $(p=0.23)$. At 12 months after surgery, bone fusion was achieved at in 22/38 patients in group B (58\%) and in 30/41 patients in group C (73\%), without significant difference between the groups $(p=0.15)$.

\section{Secondary outcomes}

VAS scores for back pain indicated that pain levels 12 months after surgery were significantly lower than preoperative levels in both groups; mean scores decreased from $5.7 \pm 1.9$ preoperatively to $2.1 \pm 0.8$ at postoperative 12 months in group $\mathrm{B}$, and from $5.5 \pm 2.4$ to $1.8 \pm 1.4$ in group $\mathrm{C}(p<0.001$ and $p=0.001$, respectively) (Table 3 ). Radiating pain intensity scoring of lower extremity 12 months after surgery also improved significantly in both groups; mean scores decreased from $7.1 \pm 2.2$ preoperatively to $1.2 \pm 1.6$

Table 2. Fusion rate on radiographs

\begin{tabular}{cccc} 
Postoperative time $(\mathrm{mo})$ & Group B $(\mathrm{n}=38)$ & Group C (n=39) & $p$-value \\
\hline 3 & $8 / 38(21)$ & $16 / 41(39)$ & 0.08 \\
6 & $18 / 38(47)$ & $25 / 41(61)$ & 0.23 \\
12 & $22 / 38(58)$ & $30 / 41(73)$ & 0.15 \\
\hline
\end{tabular}

Values are presented as number (\%). 
Table 3. Pain intensity

\begin{tabular}{|c|c|c|c|}
\hline Clinical parameter & Group B (n=38) & Group C (n=39) & $p$-value \\
\hline \multicolumn{4}{|l|}{ VAS for back pain } \\
\hline Preoperative & $5.7 \pm 1.9$ & $5.5 \pm 2.4$ & 0.58 \\
\hline 1 mo postoperative & $2.5 \pm 1.1$ & $2.2 \pm 1.3$ & 0.31 \\
\hline 3 mo postoperative & $2.2 \pm 1.0$ & $2.2 \pm 1.0$ & 0.95 \\
\hline 6 mo postoperative & $2.2 \pm 0.9$ & $2.1 \pm 1.0$ & 0.60 \\
\hline 12 mo postoperative & $2.1 \pm 0.8$ & $1.8 \pm 1.4$ & 0.21 \\
\hline \multicolumn{4}{|c|}{ VAS for radiating pain on the lower extremity } \\
\hline Preoperative & $7.1 \pm 2.2$ & $7.6 \pm 2.2$ & 0.26 \\
\hline 1 mo postoperative & $1.5 \pm 1.2$ & $1.7 \pm 1.1$ & 0.32 \\
\hline 3 mo postoperative & $1.2 \pm 1.2$ & $0.9 \pm 1.1$ & 0.38 \\
\hline 6 mo postoperative & $1.1 \pm 1.3$ & $0.9 \pm 1.2$ & 0.52 \\
\hline 12 mo postoperative & $1.2 \pm 1.6$ & $1.0 \pm 1.2$ & 0.60 \\
\hline
\end{tabular}

Values are represented as mean \pm standard deviation.

VAS, visual analogue scale.

Table 4. Neck disablility index score

\begin{tabular}{lccc} 
Clinical parameter & Group B $(\mathrm{n}=38)$ & Group C (n=39) & $p$-value \\
\hline Preoperative & $60 \pm 14$ & $59 \pm 15$ & 0.69 \\
1 mo postoperative & $36 \pm 15$ & $32 \pm 13$ & 0.24 \\
\hline 3 mo postoperative & $31 \pm 13$ & $28 \pm 13$ & 0.36 \\
6 mo postoperative & $26 \pm 13$ & $16 \pm 13$ & 0.67 \\
\hline 12 mo postoperative & $22 \pm 14$ & $21 \pm 14$ & 0.75 \\
\hline
\end{tabular}

Values are presented as mean \pm standard deviation.

Table 5. Laboratory tests of inflammatory profiles

\begin{tabular}{lccc} 
Inflammatory profiles at postoperative 2 wk & Group B $(\mathrm{n}=38)$ & Group C (n=39) & $p$-value \\
ESR $(\mathrm{mm} / \mathrm{hr})$ & $19 \pm 14$ & $17 \pm 13$ & 0.67 \\
CRP $(\mathrm{mg} / \mathrm{dL})$ & $0.24 \pm 0.55$ & $0.32 \pm 0.68$ & 0.54 \\
WBC $(103 / \mu \mathrm{L})$ & $6,250 \pm 1,940$ & $6,540 \pm 1,360$ & 0.38 \\
\hline
\end{tabular}

Values are presented as mean \pm standard deviation.

ESR, erythrocyte sedimentation rate; CRP, C-reactive protein; WBC; white blood cell count.

at postoperative 1 year in group $B$, and from $7.6 \pm 2.2$ to $1.0 \pm 1.2$ in group $C(p<0.001$ and $p<0.001$, respectively). There was no statistically significant difference between the two groups in terms of pain on the back pain and radiating pain both preoperatively and at all time points during follow-up (Table 3 ).

The mean ODI score was similar in both groups at baseline with no significant difference $60 \pm 14$ in group $\mathrm{B}$ and $59 \pm 15$ in group C $(p=0.69)$ (Table 4$)$. Patients in both groups showed marked improvement in ODI scores from baseline at 1-year follow-up time; $22 \pm 14$ in group $B$ and $21 \pm 14$ in group C. There was no statistically significant difference between the two groups in ODI score preoperatively as well as at all time points during followup (Table 4).

Two weeks after surgery, laboratory examination of inflammatory profiles revealed that ESR was $19 \pm 14 \mathrm{~mm} / \mathrm{hr}$, CRP was $0.24 \pm 0.55 \mathrm{mg} / \mathrm{dL}$, and $\mathrm{WBC}$ was $(6,250 \pm$ 
$1,940) \times 10^{3} / \mu \mathrm{L}$ in group B, and ESR was $17 \pm 13 \mathrm{~mm} / \mathrm{hr}$, CRP was $0.32 \pm 0.68 \mathrm{mg} / \mathrm{dL}$, and $\operatorname{WBC}(6,540 \pm 1,360) \times 10^{3} /$ $\mu \mathrm{L}$ in group $\mathrm{C}$ (Table 5 ). There were no statistically significant differences $(p=0.67, p=0.54$, and $p=0.38$, respectively). There were no patients with postoperative infection in either group.

\section{Discussion}

Fusion status has been an important prognostic factor in PLF $[2,3]$. Although an autograft can achieve a high union rate, autograft harvest-related morbidity makes allograft or synthetic materials alternative options [4-7].

The use of local bone graft from laminectomy and facetectomy sites during decompression has gained popularity in recent years [14]. Local bone graft alone in PLF can achieve good radiological and clinical outcomes [15-17]. The one significant disadvantage in the use of local bone graft is the limited supply that depends on the spinal levels decompressed [14].

DBM has different properties than graft substitutes that include HA. DBM is osteoinductive mainly due to the presence of low dose of bone morphogenic protein $(\sim 0.1 \%$ by weight) [18]. In addition to its osteoinductivity, DBM has osteoconductivity because collagenous and noncollagenous proteins serve as osteoconductive material that is left after the demineralization process [19]. No human studies of the use of DBM alone in PLF have been reported [15]. In a canine study, DBM alone was ineffective in achieving stable posterior spinal fusions [20]. In a controlled rabbit study, DBM was implicated as a bone graft extender but not as a pure substitute or enhancer [21]. DBM functioned as an adequate graft extender for PLF and promoted adequate fusion when mixed with a small amount of autogenous bone graft in prospective randomized study [22].

$\mathrm{HA}$ is osteoconductive but is not osteoinductive $[4,7,8]$. Although HA is useful as a bone graft substitute in posterior scoliosis surgery [23] and anterior cervical fusion [24], HA proved inadequate for PLF as stand-alone graft in a prospective matched case study [25]. The intertransverse area is a difficult region to obtain solid fusion when compared with intraosseous regions and the filling of bone defects [26]. Acharya et al. [25] reported HA underwent resorption without the formation of bridging callus in PLF. However, HA in combination with autologous bone for PLF can be used safely and effectively [27].
Based on the collective anatomical and biomechanical data, we used DBM and HA as bone graft extender, but not as a pure bone substitute because of the risk of nonunion.

In the near-absence of clinical data, we hypothesized that a material that is both osteoinductive and osteoconductive as a bone graft extender could provide better fusion outcomes for PLF than a material with osteocondutivity alone. A MEDLINE search identified only one report on the comparison study of osteoconductive and osteoinductive bone graft substitutes for lumbar spine fusion. The study grouped seven different bone graft substitutes into two groups according to their osteoinductivity or osteoconductivity and retrospectively analyzed 126 patients [28]. Our study is the first to compare the outcomes of DBM and HA as bone graft extenders for PLF.

DBM did not provide a superior outcomes in terms of fusion rate and clinical outcomes compared with $\mathrm{HA}$. Fusion rates were not significantly different between the two groups. Clinically, there were no statistically significant differences between the two groups in terms of improvement in pain intensity, ODI score, postoperative inflammatory profiles, and infection rate.

Under physiologic conditions, HA is resorbed very slowly. HA is a radio-opaque material. It may be difficult to distinguish bone from residual synthetic calcium phosphates when radiographs are used as outcome measures in fusion studies. Although statistically insignificant, the fusion rates for $\mathrm{HA}$ at 3, 6, and 12 months were higher than those of DBM. The rates may have been affected by the radio-opacity of $\mathrm{HA}$.

In the current study, the fusion rate was slightly lower than in previous reports. This likely reflected our very strict criteria of bone union. We assessed both functional and structural integrity of fusions. The measurement with dynamic radiographs was strictly carried out for definite evaluation of the fusion status. We defined pseudarthrosis as angular motion $<2^{\circ}$ or $2 \mathrm{~mm}$ of sagittal motion in Cobb angles on post-operative flexion-extension radiographs. Our radiographic criterion is stricter than the Food and Drug Administration regulation, in which fusion is defined as angular motion $<5^{\circ}$ and $<3 \mathrm{~mm}$ of sagittal motion on dynamic radiographs [29]. Because the instrumentation used in this study might have inhibited motion on dynamic radiographs, we checked fusion mass additionally. In patients who had more than single-level fusion, each level was not evaluated independently and the entire 
levels had to show continuity in the fusion mass to be considered as arthrodesis. Even the existence of singlelevel pseudarthrosis was regarded as totally unfused.

We did not perform computed tomography (CT) scans to access the status of spinal fusion. Despite improved accuracy, CT scanning has significant limitations such as cost and radiation exposure. Thin-cut CT may be less sensitive in cases of instrumented fusion in which metallic artifact obscures the fusion. Performing CT scans even for asymptomatic patients may create an ethical problem.

This study has several limitations. First, even though the clinical and radiographic data were prospectively collected, the study is retrospective in nature. Second, this study was performed without statistical power analysis and likely inadequate small sample size. Therefore, a prospective study design with power analysis might be able to better compare the clinical and radiological outcomes.

\section{Conclusions}

DBM was evaluated as a bone graft extender for PLF with $\mathrm{HA}$ in a retrospective design. There was no statistically significant difference between the fusion materials in union rate, which was the primary outcome. In addition, there were no significant differences in the secondary outcomes of pain at the back and lower extremities, ODI score, laboratory tests of inflammatory profiles, and infection rate. Based on the results, it can be concluded that DBM as a bone graft extender for PLF provides noninferior outcomes compared to the HA and could be a notable alternative.

\section{Conflict of Interest}

No potential conflict of interest relevant to this article was reported.

\section{References}

1. Yuan HA, Garfin SR, Dickman CA, Mardjetko SM. A historical cohort study of pedicle screw fixation in thoracic, lumbar, and sacral spinal fusions. Spine (Phila Pa 1976) 1994;19:2279S-2296S.

2. Kornblum MB, Fischgrund JS, Herkowitz HN, Abraham DA, Berkower DL, Ditkoff JS. Degenerative lumbar spondylolisthesis with spinal stenosis: a prospective long-term study comparing fusion and pseudarthrosis. Spine (Phila Pa 1976) 2004;29:72633.

3. Tsutsumimoto T, Shimogata M, Yoshimura Y, Misawa $H$. Union versus nonunion after posterolateral lumbar fusion: a comparison of long-term surgical outcomes in patients with degenerative lumbar spondylolisthesis. Eur Spine J 2008;17:1107-12.

4. Fischer CR, Cassilly R, Cantor W, Edusei E, Hammouri Q, Errico T. A systematic review of comparative studies on bone graft alternatives for common spine fusion procedures. Eur Spine J 2013;22:142335.

5. Younger EM, Chapman MW. Morbidity at bone graft donor sites. J Orthop Trauma 1989;3:192-5.

6. Laurie SW, Kaban LB, Mulliken JB, Murray JE. Donor-site morbidity after harvesting rib and iliac bone. Plast Reconstr Surg 1984;73:933-8.

7. Giannoudis PV, Dinopoulos H, Tsiridis E. Bone substitutes: an update. Injury 2005;36 Suppl 3:S20-7.

8. Vaccaro AR, Chiba K, Heller JG, et al. Bone grafting alternatives in spinal surgery. Spine J 2002;2:206-15.

9. Zimmermann G, Moghaddam A. Allograft bone matrix versus synthetic bone graft substitutes. Injury 2011;42 Suppl 2:S16-21.

10. Benglis DM, Boden SD, Wang MY. Biology of spine fusion. In: Benzel EC, editor. Spine surgery: techniques, complication avoidance, and management. 3rd ed. Philadelphia: Elsevier Saunders; 2012. p.14554.

11. Yi J, Lee GW, Nam WD, et al. A prospective randomized clinical trial comparing bone union rate following anterior cervical discectomy and fusion using a polyetheretherketone cage: hydroxyapatite/Btricalcium phosphate mixture versus hydroxyapatite/ demineralized bone matrix mixture. Asian Spine J 2015;9:30-8.

12. Lee KJ, Roper JG, Wang JC. Demineralized bone matrix and spinal arthrodesis. Spine J 2005;5(6 Suppl):217S-223S.

13. Fischgrund JS, Mackay M, Herkowitz HN, Brower R, Montgomery DM, Kurz LT. 1997 Volvo Award winner in clinical studies: degenerative lumbar spondylolisthesis with spinal stenosis: a prospective, randomized study comparing decompressive laminectomy and arthrodesis with and without spinal instrumentation. Spine (Phila Pa 1976) 1997;22:280712. 
14. Hsu WK, Nickoli MS, Wang JC, et al. Improving the clinical evidence of bone graft substitute technology in lumbar spine surgery. Global Spine J 2012;2:23948.

15. Sengupta DK, Truumees E, Patel CK, et al. Outcome of local bone versus autogenous iliac crest bone graft in the instrumented posterolateral fusion of the lumbar spine. Spine (Phila Pa 1976) 2006;31:985-91.

16. Ohtori S, Koshi T, Suzuki M, et al. Uni- and bilateral instrumented posterolateral fusion of the lumbar spine with local bone grafting: a prospective study with a 2-year follow-up. Spine (Phila Pa 1976) 2011; 36:E1744-8.

17. Inage $\mathrm{K}$, Ohtori $\mathrm{S}$, Koshi $\mathrm{T}$, et al. One, two-, and three-level instrumented posterolateral fusion of the lumbar spine with a local bone graft: a prospective study with a 2-year follow-up. Spine (Phila Pa 1976) 2011;36:1392-6.

18. Kwon B, Jenis LG. Carrier materials for spinal fusion. Spine J 2005;5(6 Suppl):224S-230S.

19. Martin GJ Jr, Boden SD, Titus L, Scarborough NL. New formulations of demineralized bone matrix as a more effective graft alternative in experimental posterolateral lumbar spine arthrodesis. Spine (Phila Pa 1976) 1999;24:637-45.

20. Cook SD, Dalton JE, Prewett AB, Whitecloud TS 3rd. In vivo evaluation of demineralized bone matrix as a bone graft substitute for posterior spinal fusion. Spine (Phila Pa 1976) 1995;20:877-86.

21. Morone MA, Boden SD. Experimental posterolateral lumbar spinal fusion with a demineralized bone matrix gel. Spine (Phila Pa 1976) 1998;23:159-67.

22. Cammisa FP Jr, Lowery G, Garfin SR, et al. Twoyear fusion rate equivalency between Grafton DBM gel and autograft in posterolateral spine fusion: a prospective controlled trial employing a side-byside comparison in the same patient. Spine (Phila Pa 1976) 2004;29:660-6.

23. Ransford AO, Morley T, Edgar MA, et al. Synthetic porous ceramic compared with autograft in scoliosis surgery. A prospective, randomized study of 341 patients. J Bone Joint Surg Br 1998;80:13-8.

24. Thalgott JS, Fritts K, Giuffre JM, Timlin M. Anterior interbody fusion of the cervical spine with coralline hydroxyapatite. Spine (Phila Pa 1976) 1999;24:12959.

25. Acharya NK, Kumar RJ, Varma HK, Menon VK. Hydroxyapatite-bioactive glass ceramic composite as stand-alone graft substitute for posterolateral fusion of lumbar spine: a prospective, matched, and controlled study. J Spinal Disord Tech 2008;21:106-11.

26. Boden SD, Martin GJ Jr, Morone M, Ugbo JL, Titus L, Hutton WC. The use of coralline hydroxyapatite with bone marrow, autogenous bone graft, or osteoinductive bone protein extract for posterolateral lumbar spine fusion. Spine (Phila Pa 1976) 1999;24:320-7.

27. Fujibayashi S, Shikata J, Tanaka C, Matsushita M, Nakamura T. Lumbar posterolateral fusion with biphasic calcium phosphate ceramic. J Spinal Disord 2001;14:214-21.

28. Kurd M, Cohick S, Park A, Ahmadinia K, Lee J, An H. Fusion in degenerative spondylolisthesis: comparison of osteoconductive and osteoinductive bone graft substitutes. Eur Spine J 2015;24:1066-73.

29. U.S. Department of Health and Human Services, Food and Drug Administration Center for Devices and Radiological Health. Guidance document for the preparation of IDEs for spinal systems. Rockville: Food and Drug Administration Center for Devices and Radiological Health; 2000. 